\title{
VROBOTATOR: A VIRTUAL ROBOT FACILITATOR OF SMALL GROUP DISCUSSIONS FOR K-12
}

\author{
Elinor Mizrahi, Noa Danzig and Goren Gordon ${ }^{1 *}$ \\ Curiosity Lab, Department of Industrial Engineering, Tel-Aviv University, Israel
}

\begin{abstract}
The COVID-19 pandemic has stressed the importance of efficient and accommodating online educational experiences. In this contribution, we present work-in-progress on the development of a novel system for facilitation of small group online discussions using an avatar during video conferencing. Four groups of eighth and ninth grades' students interacted with the system, with and without avatar guidance. Our pilot study results show that students reported the avatar-guided learning to be more efficient, easier to follow, and inducing more engagement and active participation. Students also showed better understanding of the learned subject with the avatar guidance, as shown by the post-session questionnaires. This system shows promise in future online educational activities as a facilitator of discussions with K-12 students.
\end{abstract}

\section{KEYWORDS}

Virtual Agent, Group Activity, Video Conference, Facilitation

\section{INTRODUCTION}

Prior to the COVID-19 pandemic, online learning environments for K-12 have not been a mainstream methodology. However, the pandemic has revealed the importance, as well as the advantages and drawbacks of video conferencing technologies, as well as other social educational technologies.

In this short paper, we introduce a system we developed and started testing during the pandemic. The system aims to facilitate small online group discussions during video conferencing. It introduces an avatar, a virtual agent, that guides the students through a learning module as well as facilitates group discussions. The content for the learning modules was created by teachers using an LMS (Learning Management System) we designed for this purpose. The students interact with the system through a dedicated website, which embeds a Zoom live video chat, a virtual agent and an interactive content area.

In a pilot study, four groups interacted with the system. Each group completed two learning modules, one with the avatar guidance and one without guidance. Of the four groups, two groups of eight-grade students learned a science unit, and two groups of ninth-grade students learned a literature unit. Questionnaires assessing both the students' experience with the system and their understanding of the learned material were administered after completing each module. The system also computed speech detection and facial expression recognition in real-time.

Our preliminary results show that: (i) the students preferred the avatar-guided module on all scales, e.g. they reported they listened more to their group members and learned more; (ii) they learned better with the avatar guidance and ;(iii) they engaged more in the discussion and actively participated more with the avatar guidance.

This pilot study shows the promise of a new online system to promote students for better discussion skills and advancing their learning in an online environment, which is predicted to become more prevalent in the post COVID era.

\footnotetext{
$1 *$ Corr. Author: goren@ gorengordon.com
} 


\section{RELATED WORK}

Small group learning activities have been considered as a promising education format (Johnson and Johnson 2009). Small-group activities can enhance student thinking and learning of both formal (e.g., math) and informal (e.g., appropriate social skills, motivated student engagement) content and skills, e.g., (Elias and Schwab 2006; Webb 1995; Hadwin, Järvelä, and Miller 2018). Moreover, children who participated in structured group activities have been shown to be more willing to work with others on the assigned tasks and they provided more elaborate help and assistance to each other than their peers in the unstructured groups (Gillies 2004).

While on-line discussion forums have prospered in recent years (Chiu and Hew 2018; Pendry and Salvatore 2015; Yang et al. 2015), with artificial intelligence (AI) assisting in managing such forums (Goel and Joyner 2017), studies have shown that personal face-to-face interactions and discussions in small groups have their advantages (Chen and Chen 2015; Thomas and Thorpe 2019). The question of scaling-up group facilitation is, thus, of prominent importance. Acquiring observational data during group activities is important to better understand the group dynamics (Vriesema and McCaslin 2020), and is easier when the facilitator is a virtual agent with full information.

The appearance of virtual visual agents, avatars, has also initiated a wider usage of conversational and social aspects of interaction. While a face of a conversational agent has been shown to increase rapport and other participant-agent related measures (Shamekhi et al. 2018), it remains an open question of what educational benefits such an avatar presents.

\section{METHODS}

THE SYSTEM. The system includes two web applications composed of multiple sub-systems. The first web application is an LMS (Learning Management System), which allows teachers and instructional designers to create learning modules containing interactive content. A module is composed of blocks, each containing content sections that either appear on the screen, e.g. multiple-choice questions, reading material; or delivered orally by the avatar. A block also contains several options allowing to prompt a facilitated discussion following the content delivery.

The second web application, and the focus of this report, is the student-centered platform, with which the students interact. The application embeds (see Figure 1: left): (a) a robotic avatar from SitePal.com; (b) a frame with the Zoom web API, that enables gallery view to see all the group members in a live chat, and; (c) an interactive content panel, displaying the content created in the LMS.
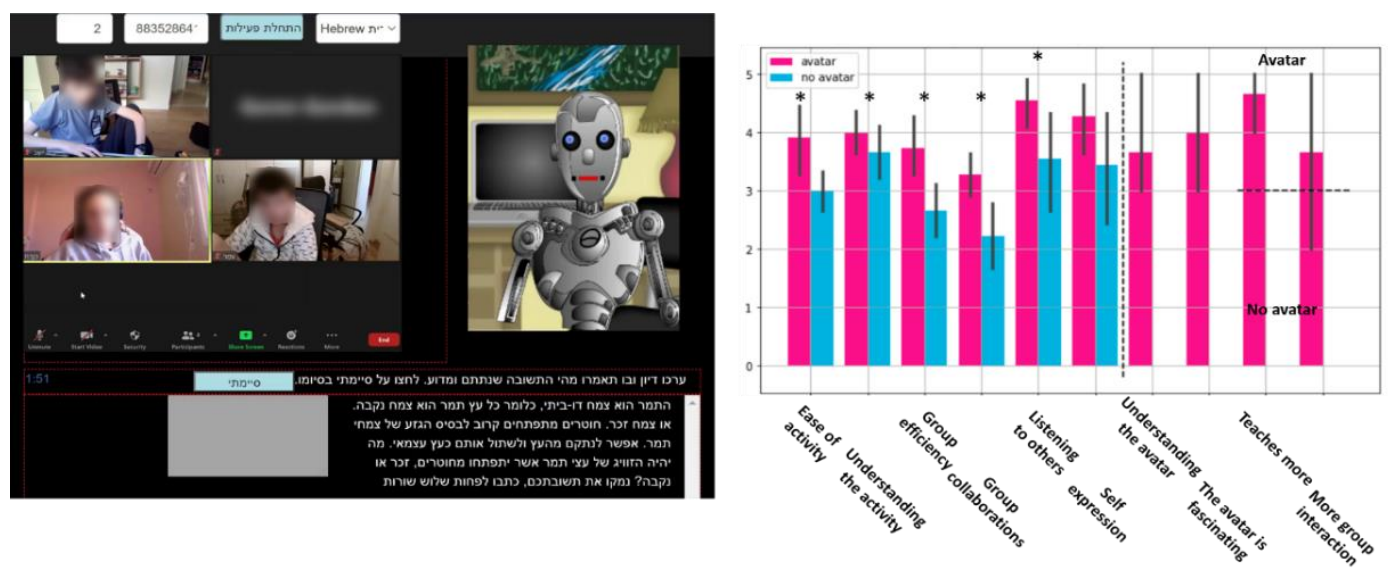

Figure 1. Left: A screenshot of the system, with (left) a Zoom API, (right) SitePal.com robotic avatar and (bottom) interactive content panel displaying a passage followed by an open-ended question, and a text-box for typing the answer. Right: Results of a 5-point semantic differential scale on various questions after the activity with and without the avatar. $* \mathrm{p}<0.05$, Wilcoxon signed-ranked test 
During a learning session, the students are individually presented with the interactive content created by the teacher in the LMS, followed by a group discussion facilitated by the avatar. A student can click on a "done" button in the interactive panel to inform the system a task is finished, so the learning session can continue to the next section.

The system is equipped with perceptual sensors (Figure 2:left). Using face-api.js, a client-side facial expression recognition system was activated, that enabled real-time and continuous analysis of facial expression such as happy and angry. A sound volume-meter was activated on the client-side, in order to detect speech in real-time.

System Architecture
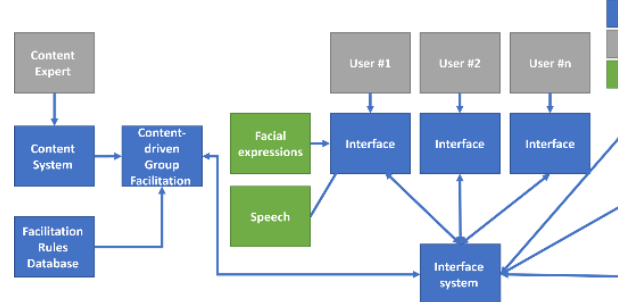

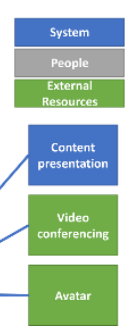

Study flow

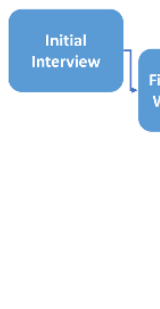

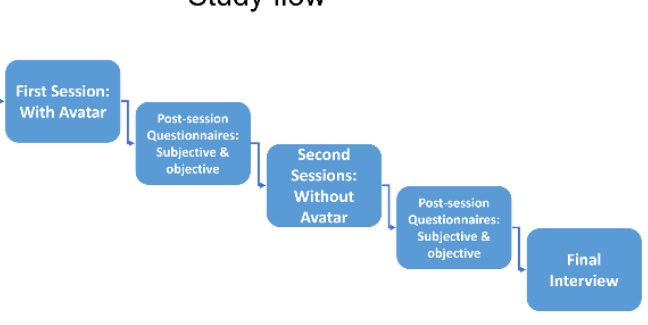

Figure 2. System architecture and study flow

The avatar was programmed with behaviors that reflect group facilitation best practices. An expert group facilitator aided in formalizing the appropriate system behaviors, e.g. giving feedback and dealing with objections, which were then programmed into the avatar. Each behavior was formulated as trigger/event pairs. For example, personal feedback was formulated as: trigger - whether the system was in a state after a question with a discussion, and whether there is a student who has not yet received positive feedback. If the condition is met, the action will be performed at the end of the question / discussion in the form of a single positive feedback to the student, out of the following types: correct answer ("great job, David, you have answered correctly"), correct and quick answer ("great job, David, you answered correctly and fast"), participation in a discussion or answering a question ("thank you, David, for contributing to the discussion"). Another example is the behavior to initiate the discussion, by addressing students. Therefore, the trigger, for example, is if all the students answered wrong answers and a discussion begins; and the action is that the facilitator addresses the student who spoke the least.

PARTICIPANTS. The study was conducted on eighth and ninth grades students, in a single middle school. Out of the eighth-grade participant pool, 20 participants (12 males, 8 females) were recruited, but due to technical difficulties such as slow internet connection and lack of camera and/or microphone, only 5 remained ( 3 males and 2 females). The participants were divided into 2 groups: (a) 3 participants ( 2 males and 1 female), (b) 2 participants ( 1 male and 1 female). The two groups each completed two learning modules in science (cell biology unit).

Out of the ninth-grade participant pool, 15 participants (11 males and 4 females) were recruited. Due to similar technical difficulties, 9 students were excluded. The participants were divided into 2 groups: (a) 3 participants ( 3 males), (b) 3 participants ( 2 males and 1 female.) The two groups each completed two learning modules in literature.

All participants' parents signed a consent form. The study was approved by the institutional IRB and the ministry of education.

CONDITIONS \& MEASURES. Each group interacted with the system twice, completing two learning modules - one facilitated by the avatar and one non-facilitated.

While a learning module content stay the same whether the avatar is present or not, an avatar-facilitated learning experience includes oral instructions, direct address to group members and various types of feedback given by the avatar, in addition to written instructions on the interactive content panel. A non-facilitated learning experience includes written instructions only, and the avatar is not shown at all on the students' screen.

During the non-facilitated parts, we confirmed with each group that they indeed saw and understood the written instructions, e.g. start a discussion. However, we did not interfere or facilitate the discussion in any way. 
The study was designed to have two types of measures: subjective, self-report; and objective, perceptual. After each activity, each student filled two types of online questionnaires: the first was composed of eight 5-point semantic differential scale questions regarding the activity and two rating questions on a scale of 1-5 comparing the avatar-facilitated experience to the non-facilitated experience, and the second was composed of four true/false questions based on the material covered in the learning module. Furthermore, the perceptual part of the system supplied information regarding when each student spoke and a continuous stream of facial expressions.

\section{RESULTS}

Questionnaires: Students reported (Figure 1: right) that the avatar-guided learning session was easier, induced a more efficient group interaction and collaboration, compared to the non-guided session. Furthermore, they reported to have listened more to their group members as well as expressing themselves more with the avatar guidance, compared to the non-guided session. Finally, the avatar was rated to be fascinating and easy to follow, and when asked to compare it directly to the non-guided session, students reported that the avatar-guided session induced more effective learning and more group interaction.

Learning: Students interacting with the avatar answered more material-related questions correctly, compared to the a learning session without the avatar, Figure 3: right-top.

Dynamics: An example of the continuous perceptual data can be seen in Figure 3: left, where the avatar's prompting was followed by more discussion, especially by the individual students it addressed.

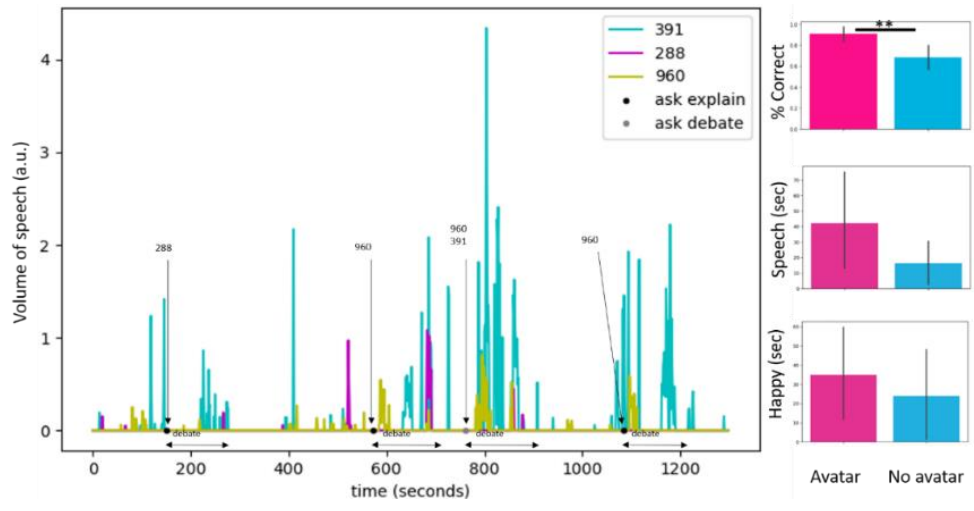

Figure 3. Left: Dynamics of interaction, as measured by the volume of speech per participant. The avatar's prompting is also presented. Right: mean over students per condition (error bars represent standard-deviation). Top: Percent of correct answer (out of eight true/false questions), four after an avatar-guided session and four after a non-guided session. Middle: cumulative time of speech. Bottom: Cumulative time of happy expression. ${ }^{* *} \mathrm{p}<0.01$, Wilcoxon signed-ranked test

\section{DISCUSSION AND CONCLUSIONS}

This short paper introduces a work-in-progress of a novel system that facilitates small group on-line discussion using video conferencing and group-facilitation best practices. The pilot study had several major limitations: The first is the large number of technical difficulties, which resulted in massive loss of participants. The second is that the first session was avatar-guided while the second was non-guided, introducing a confound of order effects. Finally, the small number of participants does not enable conclusions regarding generalizations.

This study is, to the best of our knowledge, the first to have a fully autonomous virtual agent facilitating a small group discussion in school context, with curricular content. However, more studies, both in depth and in breadth, should be performed before any conclusions regarding the efficacy of the presented system can be determined. Nevertheless, the pilot study results show the promise of this novel system as a facilitator of more effective and engaging discussions and more learning in an environment that is becoming more prevalent in the formal educational system. 


\section{REFERENCES}

Chen, Yang-Hsueh, and Pin-Ju Chen. 2015. "MOOC Study Group: Facilitation Strategies, Influential Factors, and Student Perceived Gains." Computers \& Education 86 (August): 55-70. https://doi.org/10.1016/j.compedu.2015.03.008.

Chiu, Thomas K. F., and Timothy K. F. Hew. 2018. "Factors Influencing Peer Learning and Performance in MOOC Asynchronous Online Discussion Forum." Australasian Journal of Educational Technology 34(4): 16-28. https://doi.org/10.14742/ajet.3240.

Elias, Maurice J., and Yoni Schwab. 2006. "From Compliance to Responsibility: Social and Emotional Learning and Classroom Management." Handbook of Classroom Management: Research, Practice, and Contemporary Issues, 309-41.

Gillies, Robyn M. 2004. "The Effects of Cooperative Learning on Junior High School Students during Small Group Learning." Learning and Instruction 14 (2): 197-213. https://doi.org/10.1016/S0959-4752(03)00068-9.

Goel, Ashok K., and David A. Joyner. 2017. "Using AI to Teach AI: Lessons from an Online AI Class.” AI Magazine 38 (2): 48-59. https://doi.org/10.1609/aimag.v38i2.2732.

Hadwin, Allyson, Sanna Järvelä, and Mariel Miller. 2018. "Self-Regulation, Co-Regulation, and Shared Regulation in Collaborative Learning Environments." In Handbook of Self-Regulation of Learning and Performance, 2nd Ed, 83-106. Educational Psychology Handbook Series. New York, NY, US: Routledge/Taylor \& Francis Group.

Johnson, David W., and Roger T. Johnson. 2009. “An Educational Psychology Success Story: Social Interdependence Theory and Cooperative Learning:” Educational Researcher, June. https://doi.org/10.3102/0013189X09339057.

Pendry, Louise F., and Jessica Salvatore. 2015. "Individual and Social Benefits of Online Discussion Forums." Computers in Human Behavior 50 (September): 211-20. https://doi.org/10.1016/j.chb.2015.03.067.

Shamekhi, Ameneh, Q. Vera Liao, Dakuo Wang, Rachel K. E. Bellamy, and Thomas Erickson. 2018. "Face Value? Exploring the Effects of Embodiment for a Group Facilitation Agent." In Proceedings of the 2018 CHI Conference on Human Factors in Computing Systems, 1-13. CHI '18. New York, NY, USA: Association for Computing Machinery. https://doi.org/10.1145/3173574.3173965.

Thomas, Glyn, and Stephen Thorpe. 2019. "Enhancing the Facilitation of Online Groups in Higher Education: A Review of the Literature on Face-to-Face and Online Group-Facilitation." Interactive Learning Environments 27 (1): 62-71. https://doi.org/10.1080/10494820.2018.1451897.

Vriesema, Christine Calderon, and Mary McCaslin. 2020. "Experience and Meaning in Small-Group Contexts: Fusing Observational and Self-Report Data to Capture Self and Other Dynamics." Frontline Learning Research 8 (3): 126-39. https://doi.org/10.14786/flr.v8i3.493.

Webb, Noreen M. 1995. "Learning in Small Groups." Interaction in Cooperative Groups: The Theoretical Anatomy of Group Learning, 102.

Yang, Diyi, Miaomiao Wen, Iris Howley, Robert Kraut, and Carolyn Rose. 2015. "Exploring the Effect of Confusion in Discussion Forums of Massive Open Online Courses." In Proceedings of the Second (2015) ACM Conference on Learning @Scale, 121-30. L@S '15. New York, NY, USA: ACM. https://doi.org/10.1145/2724660.2724677. 